\title{
Dentinmatrixproteine und die regenerative Endodontie
}

\begin{abstract}
Auch 2015 förderte die DGR ${ }^{2} Z$ und das Dentalunternehmen GC mit der DGR ${ }^{2} Z-G C$-Forschungsförderung experimentelle oder klinische Forschungsvorhaben im Bereich der regenerativen Zahnerhaltung - insbesondere auf dem Gebiet Restaurative Zahnheilkunde und zur Zahnhartgeweberegeneration. Die feierliche Preisübergabe erfolgte auf der Gemeinschaftstagung von DGZ, DGET, DGPZM und DGR ${ }^{2} Z$ im November in München. Wir unterhielten uns mit dem aktuellen Preisträger der DGR ZZ-GC-Forschungsförderung, Dr. Matthias Widbiller, über sein geplantes Forschungsprojekt „Die Wirkung von Dentinmatrixproteinen auf humane Pulpazellen und deren Bedeutung für die regenerative Endodontie“. Widbiller verspricht sich Erkenntnisse über Dentinmatrixproteine, die eine wichtige Rolle für den positiven Therapieerfolg regenerativer endodontischer Verfahren spielen könnten.
\end{abstract}

\begin{abstract}
? Herr Dr. Widbiller, herzlichen Glückwunsch zum Gewinn der DGR ${ }^{2} Z$-GCForschungsförderung. Wie sind Sie auf diesen Förderpreis gestoßen?

Widbiller: Im Rahmen meiner Assistentenstelle in der Poliklinik für Zahnerhaltung und Parodontologie an der Universitätsklinik Regensburg betreue ich den Behandlungskurs I der Zahnerhaltung und Parodontologie mit - gleichzeitig bin ich dort wissenschaftlich und zahnärztlich tätig. In unserer wöchentlichen Assistentenbesprechung teilte Prof. Dr. Wolfgang Buchalla die Flyer zur Forschungsförderung der $D G R^{2} Z$ aus. Weitere Informationen erhielt ich von unserer Oberärztin Frau Prof. Dr. Kerstin Galler.
\end{abstract}

Können Sie Ihr Forschungsprojekt näher erläutern?

Widbiller: Es ist bekannt, dass Odontoblasten während der Zahnbildung Wachstumsfaktoren sowie andere bioaktive Proteine sezernieren und diese in Dentin einbetten, wo sie zeitlebens in konservierter Form vorliegen. Diese bioaktiven Proteine können mithilfe demineralisierender Substanzen wie EDTA, Kalziumhydroxid etc. freigesetzt und isoliert werden. Als autologe Signalmoleküle sind sie in der Lage, das Zellverhalten bei Maßnahmen zum Vitalerhalt der Zahnpulpa oder im Zuge regenerativer endodontischer Verfahren zu beeinflussen. Daher ist das Ziel des nun geförderten Projekts, klinisch relevante Erkenntnisse über die Effekte von Dentinmatrixproteinen auf humane Pulpazellen zu gewinnen. Im Einzelnen möchten wir den
Einfluss auf die Zellmigration, den Effekt auf die Proliferation und Vitalität, ihre mögliche Induktion von Apoptose und $\mathrm{Ne}$ krose und die Auswirkungen auf die Mineralisation sowie die Expression mineralisationsassoziierter Gene untersuchen.

? Wie setzen Sie dieses Vorhaben konkret um?

Widbiller: In der praktischen Umsetzung werde ich ein speziell entwickeltes Verfahren nutzen, mit dem die Matrixproteine aus Dentinmehl isoliert, gereinigt und konzentriert werden. Anschließend lassen sich die Menge des Wachstumsfaktors TGFß-1, welcher im Dentin mengenmäßig am stärksten repräsentiert ist, sowie weitere Proteine mit einem Enzyme Linked Immunosorbent Assay (ELISA) bestimmen. Es schließen sich verschiedene In-vitro-Versuche mit den Matrixproteinen aus menschlichem Dentin in unterschiedlichen Konzentrationen mit humanen primären Pulpazellen an. Diese Versuche sind zum Beispiel Zellzahlbestimmungen mittels DNA-Quantifizierung, Vitalitätstests oder Alizarin-Färbungen. Die Dauer meiner Untersuchungen veranschlage ich mit 12 Monaten.

Welche Bedeutung könnten Ihre Erkenntnisse für die tägliche Praxis haben?

Widbiller: Regenerative endodontische Verfahren gewinnen in unserem Fachbereich zunehmend an Bedeutung und eröffnen Zahnärzten völlig neue Therapie- möglichkeiten, die bei jungen Patienten trotz Pulpanekrose einen Abschluss des Wurzelwachstums ermöglichen. Die erwarteten Ergebnisse leisten einen wichtigen Beitrag in der Erforschung der Verfahren, denn isolierte Dentinmatrixproteine

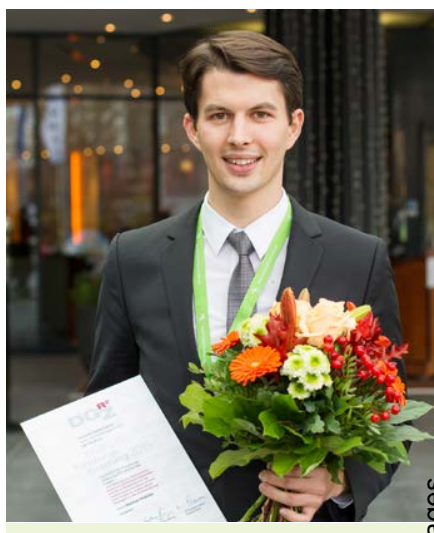
Dr. Matthias Widbiller bei der Verlei- $\frac{\pi}{\frac{\pi}{2}}$

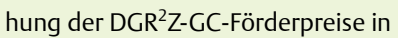
München (Foto: GC).

könnten als autologer Zusatz zu Trägermaterialien bei regenerativen endodontischen Verfahren den Therapieerfolg positiv beeinflussen.

? Wie wichtig ist die Fördersumme des DGR ${ }^{2} Z-G C-W i s s e n s c h a f t s f o n d s$ für die Realisation Ihres Vorhabens?

Widbiller: Die Fördersumme von $20000 €$ ermöglicht mir die Finanzierung aller Materialien und Reagenzien, die zur Durchführung der Experimente benötigt werden.

Wie beurteilen Sie das Engagement von Dentalunternehmen wie GC zur Wissenschaftsförderung?

Widbiller: Ich finde es großartig, dass Unternehmen wie GC gemeinsam mit Fachgesellschaften junge Wissenschaftler in der Zahnmedizin fördern und so, unabhängig von Produktangeboten, die Grundlagenforschung an deutschen Hochschulen unterstützen.

? Haben Sie Erfahrungen mit dem Produktportfolio von GC und wenn ja, wie beurteilen Sie dieses?

Widbiller: Glasionomerzemente von GC sind sowohl im Studentenkurs als auch in meinem eigenen Behandlungsalltag ein fester Bestandteil. Zudem arbeite ich sehr gerne mit Produkten der everStick-Familie, die mir die Anfertigung minimalinvasiver Restaurationen ermöglichen.

Vielen Dank für das Gespräch, wir wünschen Ihnen für die Umsetzung Ihres Forschungsprojekts viel Erfolg!

Aus einer Pressmitteilung der GC Germany GmbH, Bad Homburg 